\title{
STROKE AND CARDIAC CELL DEATH: TWO PEAS IN A POD
}

Authors: Chiara Gonzales-Portillo ${ }^{1}$, Hiroto Ishikawa ${ }^{1}$, Kazutaka Shinozuka $^{1}$, Naoki

Tajiri $^{1}$, Yuji Kaneko ${ }^{1}$, and Cesar V. Borlongan ${ }^{1 *}$

${ }^{1}$ University of South Florida, Department of Neurosurgery and Brain Repair, Tampa, FL, USA

Running Head: A Pathological Link Between Stroke and Heart Failure

\author{
*Corresponding Author: \\ Dr. Cesar V. Borlongan \\ University of South Florida \\ Department of Neurosurgery \\ Tampa, FL, 33612, USA \\ cborlong@health.usf.edu
}

Acknowledgements: CVB is funded by the National Institutes of Health 1R01NS071956, the National Institutes of Health R21 1R21NS089851, the Department of Defense W81XWH-11-1-0634, and VA Merit Review. 


\begin{abstract}
A close pathological link between stroke brain and heart failure may exist. Here, we discuss relevant laboratory and clinical reports demonstrating neural and cardiac myocyte cell death following ischemic stroke. Although various overlapping risk factors exist between cerebrovascular incidents and cardiac incidents, stroke therapy has largely neglected the cardiac pathological consequences. Recent preclinical stroke studies have implicated an indirect cell death pathway, involving toxic molecules, that originates from the stroke brain and produces cardiac cell death. In concert, previous laboratory reports have revealed a reverse cell death cascade, in that cardiac arrest leads to ischemic cell death in the brain. A deeper understanding of the crosstalk of cell death pathways between stroke and cardiac failure will facilitate the development of novel treatments designed to arrest the global pathology of both diseases thereby improving the clinical outcomes of patients diagnosed with stroke and heart failure.
\end{abstract}

Keywords: cerebrovascular disease; cardiovascular disease; neural cell death; cardiac myocyte death; circulating toxic molecules 
Ischemic heart and cerebrovascular disease make up the two leading causes of death in the world. Overall, the United States spends a total of $\$ 260.7$ billion for both cardiac disease and ischemic stroke combined [1,2]. Predisposing factors of these two diseases include high blood pressure, high blood cholesterol levels, diabetes, obesity, and a history of cardiovascular diseases in the immediate family [3-6]. Interestingly, $2-6 \%$ of the deaths caused by cardiac origins occurred approximately 3 months after an ischemic stroke $[7,8]$. This percentage is supported by data showing that those who suffer ischemic stroke are more prone to a cardiac death than non-stroke victims. After an ischemic stroke, large amounts of cardiac enzymes and plasma catecholamines have been found in the patient's body $[7,8]$. The increase of cardiac enzymes is linked to elevated amounts of troponin and creatine phosphokinase levels, which are detected when cardiac cells are under stress and/or dying [7,8]. Additionally, the increased level of catecholamine is associated with high blood pressure, under stress condition [9-11].

Despite these various overlapping risk factors, the correlation between cerebrovascular incidents and cardiac incidents has been largely neglected in the clinic. Unfortunately, $88 \%$ of the patients with ischemic stroke have presented with cardiac symptoms up to four weeks following the stroke [12]. The damage to the insular cortex produces the highest incidence of cardiac death when compared to other brain regions [12]. Although it is recognized that the insular cortex triggers cardiac myocyte death, there is disagreement on whether it is a specific sub-region of the insular cortex or the entire brain region as the cause of death. For this reason, the involvement of the insular cortex in cardiovascular disorders remains not well established. 
Recognizing this close interaction between stroke brain and heart failure, we embarked on studying the relationship between ischemic neural cell death and cardiac myocyte compromise using both in vitro and in vivo stroke models [13]. The in vitro study employed oxygen-glucose deprivation (OGD) condition in primary rat neuronal cells (PRNCs), using the supernatant to assess the cellular changes in rat cardiac myocytes (RCMs) following the ischemic-reperfusion injury. As for the in vivo study, transient middle cerebral artery occlusion (MCAo) was induced in adult rats. Immunohistochemical analyses on the brains and hearts of stroke rats was conducted to reveal the different cell death markers. We hypothesized that the ischemic brain compromises cardiac myocytes through secretion of cell death factors. This study revealed that ischemic stroke conferred cell death signals to the heart [13]. The in vitro study revealed that the PRNCs rendered toxic effects on RCMs. Likewise, the in vivo study revealed that ischemic stroke animals exhibited RCM cell death. These results suggested to us that a close pathological link exists between ischemic stroke and cardiac arrest.

Conditions including heart failure and ischemic brain stroke are associated with major neurological disabilities around the world. Instances in which these two conditions occur simultaneously account for high mortality rates [14]. Our recent study revealed cardiac myocyte death following ischemic stroke, lending further support to the notion that ischemic stroke has the potential to produce cardiac alterations [13].

This begs the question "How does the ischemic stroke disrupt heart function?" A nonpathogenic heart automatically beats while the brain controls the heart rate, but in the instance of a stroke, a major aberrant physiological consequence ensues characterized by 
the inability of the stroke brain to control heart rhythms, suggesting that the heart is now compromised as a direct result of the stroke $[8,12]$. As noted above, the insular cortex is the brain region most closely associated with the control of heart rhythm [15]. Altogether, these observations implicate a crosstalk between the insular cortex and the heart following an ischemic stroke.

Surprisingly, our results demonstrated that the direct ischemia/reperfusion (I/R) injury to the RCMs actually had no effect on cardiac myocyte death [13]. In the in vitro study, the addition of OGD supernatant was shown to generate the massive cell death within the heart, implicating that an indirect pathway, involving stroke brain-mediated cell death signals, primarily contributed to the observed cardiac alterations. Although this seems like a solid finding, other potentially parallel cell death pathways linking the two diseases (stroke and cardiac cell death) must also be considered, including: systemic responses [6,16,17], neuro-endocrine mediated myocardial suppression [7,18], and changes in circulating endothelial progenitor cells [19].

Additional immunocytochemical analyses revealed that OGD-exposed PRNCs showed a significant time-dependent increase of positive cells against all cell death markers 24 hours after I/R injury when compared to the control [13]. Moreover, ischemic stroke robustly altered the autophagic pathway over the apoptotic and necrotic cell death mechanisms, in rendering toxic effects on the heart [13], suggesting that autophagyrelated cell death signals may principally contribute to cardiac injury.

Further examination of the in vivo results revealed that the brain and heart also exhibited all the death cell markers of autophagy, necrosis, and apoptosis [13]. Of equal importance, widespread autophagic and apoptotic cells were detected not only on the 
ipsilateral side of the brain but also on the contralateral side of the brain, indicating that although the experimental stroke damage was initially limited to one hemisphere of the brain, the progression of the disease is likely to spread globally to the brain, and, as discussed above, the pathological consequences of the stroke brain may even reach the heart.

Lateralization of stroke leading to cardiac abnormalities has been advanced by Dr. Hachinski [7] and remains a hot topic for research [20]. In healthy human individuals, lateralization of cardiovascular autonomic function has been implicated in the forebrain, in particular damage to the insular cortex associated with more pronounced autonomic imbalance leading to life threatening arrhythmias and sudden death [21]. To this end, the observation that left-handers may have a lower risk of sudden death compared with righthanders [21] suggests that stroke to the right side more responsible to cardiac arrhythmias than the left. Although a similar lateralization has been suggested in insular epilepsy (e.g., dysphoric responses were statistically associated with right-sided stimulation as compared with left-sided stimulation of mesial frontal, orbitofrontal, mesial temporal, and insular stimulation sites [22]; such laterization has been challenged in the occurrence of ictal bradycardia, which most often occurs in association with bilateral hemispheric seizure activity rather than a lateralizing sign in localizing seizure onset, negating the concept of a primarily unilateral parasympathetic cardiomotor representation in the left hemisphere [23].

In major stroke, brain natriuretic peptide (BNP) can be elevated and acts as a biomarker associated with increased mortality [24]. BNP is produced by the cardiac muscle as a response to increased stress and is related with a dysfunction of the left 
ventricle and acts as a vasodilatator and natriuresis to decrease the systolic blood pressure and is also correlated with the degree of cardiac dysfunction [24]. In stroke, all-case mortality is associated with BNP as well as with N-terminal-pro-brain natriuretic peptide (NT-proBNP) and also closely coincides with cardioembolic stroke. This suggests that, in addition to the heart, the kidney is involved in stroke pathology. In addition to all-case mortality in ischemic stroke, TIA or hemorrhagic stroke is associated with an increase in BNP and NT-proBNP, suggesting a relation between stroke (pathology of the brain) and the heart. Taken together, these studies note that an increase in BNP is associated with worse outcome of stroke [25], and that renal failure is associated with increase of BNP and NT-proBNP which are both independent predictors of mortality in renal disease and may pose as risk factors for stroke in tandem with heart dysfunction.

The concept of an indirect pathological pathway of cell death originating from the brain and reaching the heart stipulates that soluble factors secreted by the ischemic brain cells may potentially be carrying cell death signals to the cardiac myocytes. The toxic molecules secreted by the ischemic brain remain to be identified. Furthermore, since these toxins travel through the circulatory system, its effects on other vital peripheral organs warrant investigations. At the very least, our study [13] suggests that following an ischemic stroke, a heart examination should be considered as part of the stroke treatment and management.

In summary, ischemic stroke may lead to cardiac cell death, paralleling a series of studies that show a complementary interaction whereby cardiac arrest results in strokelike brain pathology [14, 26-28]. The convergence of these investigations on ischemic stroke and cardiac arrest advances the notion of a pathological pathway linking the two 
diseases together. With an in-depth understanding of the molecular, cellular, and anatomical changes constantly occurring in stroke and cardiac failure, a novel treatment strategy directed at abrogating both diseases may improve the clinical outcomes of the patients suffering from these two maladies. 


\section{References}

[1] Centers for Disease Control and Prevention. Prevalence of coronary heart disease-United States, 2006-2010. MMWR Morb Mortal Wkly Rep 2011;60:1377-81.

[2] Centers for Disease Control and Prevention. Prevalence of stroke--United States, 2006-2010. MMWR Morb Mortal Wkly Rep 2012;61:379-82.

[3] Kannel WB. The Framingham Study: ITS 50-year legacy and future promise. J Atheroscler Thromb 2000;6:60-6.

[4] Lo EH, Dalkara T, Moskowitz MA. Mechanisms, challenges and opportunities in stroke. Nat Rev Neurosci 2003;4:399-415.

[5] Zhang ZG, Chopp M. Neurorestorative therapies for stroke: underlying mechanisms and translation to the clinic. Lancet Neurol 2009;8:491-500.

[6] Lawlor DA, Smith GD, Leon DA, Sterne JA, Ebrahim S. Secular trends in mortality by stroke subtype in the 20th century: a retrospective analysis. Lancet 2002;360:1818-23.

[7] Oppenheimer SM, Hachinski VC. The cardiac consequences of stroke. Neurol Clin 1992;10:167-76.

[8] Prosser J, MacGregor L, Lees KR, Diener HC, Hacke W, Davis S. Predictors of early cardiac morbidity and mortality after ischemic stroke. Stroke 2007;38:2295302.

[9] Klingelhofer J, Sander D. Cardiovascular consequences of clinical stroke. Baillieres Clin Neurol 1997;6:309-35. 
[10] Adams 3rd JE, Abendschein DR, Jaffe AS. Biochemical markers of myocardial injury. Is MB creatine kinase the choice for the 1990s? Circulation. 1993;88:75063.

[11] Snider SR, Kuchel O. Dopamine: an important neurohormone of the sympathoadrenal system. Significance of increased peripheral dopamine release for the human stress response and hypertension. Endocr Rev 1983;4:291-309.

[12] Ay H, Koroshetz WJ, Benner T, Vangel MG, Melinosky C, Arsava EM, et al. Neuroanatomic correlates of stroke-related myocardial injury. Neurology 2006;66:1325-9.

[13] Ishikawa H, Tajiri N, Vasconcellos J, Kaneko Y, Mimura O, Dezawa M, et al. Ischemic stroke brain sends indirect cell death signals to the heart. Stroke $2013 ; 44: 3175-82$.

[14] Go AS, Mozaffarian D, Roger VL, Benjamin EJ, Berry JD, Borden WB, et al. Heart disease and stroke statistics--2013 update: A report from the american heart association. Circulation 2013;127:e6-e245.

[15] Korpelainen JT, Sotaniemi KA, Myllyla VV. Autonomic nervous system disorders in stroke. Clin Auton Res 1999;9:325-33.

[16] Kostulas N, Kivisakk P, Huang Y, Matusevicius D, Kostulas V, Link H. Ischemic stroke is associated with a systemic increase of blood mononuclear cells expressing interleukin-8 mRNA. Stroke 1998;29:462-6.

[17] Mo X, Li T, Ji G, Lu W, Hu Z. Peripheral polymorphonuclear leukocyte activation as a systemic inflammatory response in ischemic stroke. Neurol Sci 2013;34:1509-16. 
[18] Wira 3rd CR, Rivers E, Silver B, Lewandowski C. The impact of cardiac contractility on cerebral blood flow in ischemia. Western J Emerg Med 2011;12:227-32.

[19] Paczkowska E, Golab-Janowska M, Bajer-Czajkowska A, Machalinska A, Ustianowski P, Rybicka M, et al. Increased circulating endothelial progenitor cells in patients with haemorrhagic and ischaemic stroke: the role of endothelin-1. J Neurol Sci 2013;325:90-9.

[20] Inamasu J, Sugimoto K, Watanabe E, Kato Y, Hirose Y. Effect of insular injury on autonomic functions in patients with ruptured middle cerebral artery aneurysms. Stroke 2013;44:3550-2.

[21] Ozdemir O, Hachinski V. Brain lateralization and sudden death: its role in the neurogenic heart syndrome. J Neurol Sci 2008;268:6-11.

[22] Smith JR, Lee GP, Fountas K, King DW, Jenkins PD. Intracranial stimulation study of lateralization of affect. Epilepsy Behav 2006;8:534-41.

[23] Britton JW, Ghearing GR, Benarroch EE, Cascino GD. The ictal bradycardia syndrome: localization and lateralization. Epilepsia 2006;47:737-44.

[24] Shibazaki K, Kimura K, Okada Y, Iguchi Y, Terasawa Y, Aoki J. Heart failure may be associated with the onset of ischemic stroke with atrial fibrillation: a brain natriuretic peptide study. J Neurol Sci 2009;281:55-7.

[25] Montaner J, Perea-Gainza M, Delgado P, Ribó M, Chacón P, Rosell A, Quintana M, Palacios ME, Molina CA, Alvarez-Sabín J. Etiologic diagnosis of ischemic stroke subtypes with plasma biomarkers. Stroke 2008;39:2280-7. 
[26] DeVries AC, Craft TK, Glasper ER, Neigh GN, Alexander JK. 2006 curt p. Richter award winner: Social influences on stress responses and health. Psychoneuroendocrinology 2007;32:587-603.

[27] Ning M, Lo EH, Ning PC, Xu SY, McMullin D, Demirjian Z, et al. The brain's heart - therapeutic opportunities for patent foramen ovale (PFO) and neurovascular disease. Pharmacol Ther 2013;139:111-23.

[28] Micheli S, Agnelli G, Caso V, Alberti A, Palmerini F, Venti M, et al. Acute myocardial infarction and heart failure in acute stroke patients: frequency and influence on clinical outcome. J Neurol 2012;259:106-10. 